\title{
METRIC SPACES IN WHICH BLUMBERG'S THEOREM HOLDS
}

\section{J. C. BRADFORD AND CASPER GOFFMAN}

H. Blumberg, [1], showed that for every real function $f$ on the real line $R$ there is a dense set $D \subset R$ such that $f$ is continuous on $D$ relative to $D$. The purpose of this paper is to characterize the metric spaces in which Blumberg's theorem holds.

Let $S$ be a metric space and $E$ a subset of $S$. A point $x \in S$ is said to be of the second category relative to $E$ if every sphere of center $x$ contains a subset of $E$ which is of the second category in $S$. Otherwise, $x$ is said to be of the first category relative to $E$. $S$ is said to be of homogeneous second category if every $x \in S$ is of the second category relative to $S$.

We prove the

THEOREM. If the metric space $S$ is of homogeneous second category then for every real function $f$ on $S$ there is a dense $D \subset S$ such that $f$ is continuous on $D$ relative to $D$.

Conversely, if $S$ is not of homogeneous second category there is a real function $f$ on $S$ which is not continuous on any dense $D \subset S$ relative to $D$.

Proof. We prove the converse first. Let $S$ be a metric space which is not of homogeneous second category. There is then a sphere $K \subset S$ which is of the first category in $S$. Let $K=\cup_{n=1}^{\infty} K_{n}$ where each $K_{n}$ is nowhere dense in $S$. Let $f(x)=n$ for every $x \in K_{n}$, and let $f(x)=0$ for every $x \in S \sim \cup_{n=1}^{\infty} K_{n}$. Let $D$ be dense in $S$ and let $x \in D \cap K$. Then $x \in K_{n}$, for some $n$, so that $f(x)=n$. Let $K^{\prime}$ be any sphere of center $x$. Since $D$ is dense and $K_{n}$ is nowhere dense there is a $y \in\left(D \sim K_{n}\right) \cap K^{\prime}$. But $|f(x)-f(y)| \geqq 1$. Hence, $f$ is not continuous on $D$ relative to $D$. By an obvious modification, the function $f$ may be taken to be bounded.

For the direct part of the theorem, we need several lemmas.

Lemma 1 (Banach, [2]). If $S$ is a metric space, ECS, and TCE is the set of points in $E$ which are of the first category relative to $E$ then $T$ is of the first category in $S$.

Let $S$ be a metric space and $E \subset S$. An $x \in S$ is said to be a heavy point relative to $E$ if there is a sphere of center $x$ every point of which is a point of the second category relative to $E$.

Received by the editors September 30, 1959. 
Lemma 2. If $S$ is a metric space and $E \subset S$, then $E=A \cup B, A \cap B$ $=\varnothing$, where every $x \in A$ is a heavy point relative to $E$ and $B$ is of the first category in $S$.

Proof. Let $B_{1}$ consist of the points of $E$ which are of the first category relative to $E$. Then $B_{1}$ is a set of the first category in $S$. Let $B_{2}$ consist of the points of $E$ which are of the second category relative to $E$ but are not heavy relative to $E$. Then every sphere in $S$ which has points of $B_{2}$ contains a subsphere which has no points of $B_{2}$, so that $B_{2}$ is nowhere dense in $S$. Then $B=B_{1} \cup B_{2}$ is of the first category in $S$ and $A=S \sim B$ is such that every $x \in A$ is contained in a sphere in $S$ every point of which is of the second category relative to $E$.

Let $f$ be a real function on a metric space $S$. We shall say that $f$ is almost continuous at $x$ if for every open set $G$ containing $f(x)$ the set $E=[y: f(y) \in G]$ has $x$ as a heavy point relative to $E$.

Lemma 3. If $S$ is a metric space and $f$ is a real function on $S$ then $f$ is almost continuous at every point of $S$ except at a set of the first category.

Proof. Let $\left\{G_{n}\right\}$ be the set of open intervals with rational end points arranged in one-one correspondence with the natural numbers. For every $n$, let $S_{n} \subset S$ be the set of points for which $f(x) \in G_{n}$ and let $E_{n} \subset S_{n}$ be the points of $S_{n}$ which are not heavy points relative to $S_{n}$. The sets $E_{n}$ are all of the first category, and so $E=\cup_{n=1}^{\infty} E_{n}$ is also of the first category. But if $f$ is not almost continuous at $x$ then $x \in E$.

Lemma 4. Let $S$ be a metric space of homogeneous second category, $f$ a real function on $S$, and $\epsilon>0$. There is a set $\mathfrak{u}=\left[U_{\alpha}: \alpha \in A\right]$ of pairwise disjoint open spheres in $S$ where, for every $\alpha \in A, U_{\alpha}$ $=\left[x: d\left(x, x_{\alpha}\right)<r_{\alpha}\right]$ with $0<r_{\alpha}<\epsilon$, and, for every $\alpha \in A$, there is a subset $V_{\alpha} \subset U_{\alpha}$, with $x_{\alpha} \in V_{\alpha}$, such that every $x \in U_{\alpha}$ is a heavy point relative to $V_{\alpha}$, with the following properties:

(a) $U_{\alpha \in A} U_{\alpha}$ is dense in $S$,

(b) for every $\alpha \in A$, and $x \in V_{\alpha},\left|f(x)-f\left(x_{\alpha}\right)\right|<\epsilon$.

Proof. Let $R \subset S$ be the set of points for which $f$ is almost continuous. By Lemma 3, and the assumption that $S$ is of homogeneous second category, $R$ is dense in $S$. Let $x_{\alpha}^{\prime}, \alpha<\xi$, be a well ordering of $R$. Let $x_{1}=x_{1}^{\prime}$, and let $U_{1}$ be a sphere of center $x_{1}$ and radius $r_{1}<\epsilon$ such that, for a subset $V_{1}$ of $U_{1}$, with $x \in V_{1}$ and with every point of $U_{1}$ of second category relative to $V_{1},\left|f(x)-f\left(x_{1}\right)\right|<\epsilon$. Let $x_{2}=x_{\alpha_{2}}^{\prime}$, where $x_{\alpha_{2}}^{\prime}$ is the first element of the well ordered set $x_{\alpha}^{\prime}, \alpha<\xi$ which 
does not belong to the closure, $\bar{U}_{1}$, of $U_{1}$. Let $U_{2}$ be a sphere of center $x_{2}$ and radius $r_{2}<\epsilon$, such that $U_{2} \cap \bar{U}_{1}=\varnothing$, and for a subset $V_{2} \subset U_{2}$, with $x_{2} \in V_{2}$, for which every point in $U_{2}$ is a point of second category relative to $V_{2},\left|f(x)-f\left(x_{2}\right)\right|<\epsilon$. Suppose, for an ordinal $\eta$, we have, for every $\beta<\eta, x_{\beta}, U_{\beta}, V_{\beta}$ such that $U_{\beta}$ is a sphere of center $x_{\beta}$ and radius $r_{\beta}<\epsilon, x_{\beta} \in V_{\beta}$, every point of $U_{\beta}$ is of second category relative to $V_{\beta},\left|f(x)-f\left(x_{\beta}\right)\right|<\epsilon$ for every $x \in V_{\beta}$, and $\mathrm{Cl}\left(\cup_{\gamma<\beta} U_{\gamma}\right) \cap U_{\beta}=\varnothing$. Let $x_{\eta}=x_{\alpha_{\eta}}^{\prime}$ be the first element of the well ordered set $x_{\alpha}^{\prime}, \alpha<\xi$, which does not belong to $\mathrm{Cl}\left(\cup_{\beta<\eta} U_{\beta}\right)$. Let $U_{\eta}$ be a sphere of center $x_{\eta}$ and radius $r_{\eta}<\epsilon$, such that $\mathrm{Cl}\left(\mathrm{U}_{\beta<\eta} U_{\beta}\right) \cap U_{\eta}=\varnothing$, and such that there is a $V_{\eta} \subset U_{\eta}, x_{\eta} \in V_{\eta}$, with every point of $U_{\eta}$ a point of second category relative to $V_{\eta}$, for which $\left|f(x)-f\left(x_{\eta}\right)\right|<\epsilon$ for every $x \in V_{\eta}$. By transfinite induction, $x_{\alpha}, U_{\alpha}, V_{\alpha}, \alpha<\zeta_{1}$, are obtained which satisfy the conditions of the lemma.

REMARK 1. For each $\alpha<\zeta_{1}, V_{\alpha}$ is itself a metric space of homogeneous second category, and so Lemma 4 holds for $V_{\alpha}$ and any $\epsilon^{\prime}>0$.

REMARK 2. For every $x \in S$ there is an $\alpha<\zeta_{1}$ with $d\left(x, x_{\alpha}\right)<2 \epsilon$.

We now prove the theorem. Let $x_{\alpha}, U_{\alpha}, V_{\alpha}, \alpha<\zeta_{1}$, be the points, spheres, and sets of Lemma 4 corresponding to $\epsilon=1$ and the metric space $S$. For every $\alpha<\zeta_{1}$, let $x_{\alpha \alpha^{1}}, U_{\alpha \alpha^{1}}, V_{\alpha \alpha^{1}}, \alpha^{1}<\zeta_{2}$, with $x_{\alpha 1}=x_{\alpha}$, be the points, spheres, and sets of Lemma 4 corresponding to $\epsilon=1 / 2$ and the metric space $V_{\alpha}$. It is possible to choose $x_{\alpha 1}=x_{\alpha}$ since $f$ is almost continuous at $x_{\alpha}$. For every $\alpha<\zeta_{1}, \alpha^{1}<\zeta_{2}$ let $x_{\alpha \alpha^{1} \alpha^{2}}, U_{\alpha \alpha^{1} \alpha^{2}}$, $V_{\alpha \alpha^{1} \alpha^{2}}, \alpha^{2}<\zeta_{3}$, with $x_{\alpha \alpha^{11}}=x_{\alpha \alpha^{1}}$, be the points, spheres, and sets of Lemma 4 corresponding to $\epsilon=1 / 3$ and the metric space $V_{\alpha \alpha^{1}}$.

We continue in this way ad infinitum, obtaining for every natural number $n, x_{\alpha \alpha^{1} \cdots \alpha^{n}}, U_{\alpha \alpha^{1} \cdots \alpha^{n}}, V_{\alpha \alpha^{1} \cdots \alpha^{n}}, \alpha^{n}<\zeta_{n+1}$, with $x_{\alpha \alpha^{1} \cdots \alpha^{n-1} 1_{1}}$ $=x_{\alpha \alpha^{\prime}} \ldots \alpha^{n-1}$. These are the points, spheres, and sets of Lemma 4 corresponding to $\epsilon=1 / n$ and the metric space $V_{\alpha \alpha^{1} \cdots \alpha^{n-1}}$. Let

$$
\begin{aligned}
& D_{0}=\left[x_{\alpha}: \alpha<\zeta_{1}\right], \quad D_{1}=\left[x_{\alpha \alpha^{1}}: \alpha<\zeta_{1}, \alpha^{1}<\zeta_{2}\right], \cdots, \\
& D_{n}=\left[x_{\alpha \alpha^{1} \cdots \alpha^{n}}: \alpha<\zeta_{1}, \alpha^{1}<\zeta_{2}, \cdots, \alpha^{n}<\zeta_{n+1}\right], \cdots,
\end{aligned}
$$

and let $D=\cup_{n=0}^{\infty} D_{n}$.

By Remark 2, $D$ is dense in $S$. Moreover, for every $n$, for every $x \in D_{n}$, the saltus of $f$ at $x$ relative to $D$ is less than $1 / n$. For, $x=x_{\alpha \alpha^{1} \ldots \alpha^{n}}$ for some $\alpha<\zeta_{1}, \alpha^{1}<\zeta_{2}, \cdots, \alpha^{n}<\zeta_{n+1}, \quad D \cap U_{\alpha \alpha^{1} \cdots \alpha^{n}}$ $\subset V_{\alpha \alpha^{1} \cdots \alpha^{n}}$ and $x \in V_{\alpha \alpha^{1} \cdots \alpha^{n}}$ implies $\left|f(x)-f\left(x_{\alpha \alpha^{1} \cdots \alpha^{n}}\right)\right|<1 / n$. Now, let $x \in D$. Then $x \in D_{n}$ for some $n$. But, evidently, $D_{0} \subset D_{1} \subset \cdots$ $\subset D_{m} \subset \cdots$, so that $x \in D_{m}$ for every $m>n$. Hence the saltus of $f$ at $x$, relative to $D$, is less than $1 / m$ for every $m$. In other words, $f$ is continuous at $x$ relative to $D$. 


\section{REFERENCES}

1. H. Blumberg, New properties of all real functions, Trans. Amer. Math. Soc. vol. 24 (1922) pp. 113-128.

2. S. Banach, Théorème sur les ensembles de première catégorie, Fund. Math. vol. 16 (1930) pp. 395-398.

\section{Abilene Christian College and \\ Purdue University}

\section{CORRECTION TO "GALOIS EXTENSIONS"}

\section{CARL FAITH}

$\S 4$ of [1] is devoted to the question of the existence of a normal basis of a certain Galois extension $\Re / \mathfrak{F}$. Theorem 2 of this section is incorrectly stated. It can be corrected by replacing the condition (3) the centralizer $\mathfrak{F}^{\prime}$ of $\mathfrak{F}$ in $\Re$ is simple by the condition $\left(3^{\prime}\right)$ the subring $V(\mathbb{S})$ generated by all regular $x \in \Omega$ effecting inner automorphisms belonging to $B$ is simple. Simplicity of $V(\mathbb{S})$ (rather than simplicity of $\left.\mathfrak{F}^{\prime}\right)$, together with the original conditions (1) and (2), implies the equality $V(\mathfrak{S})=\mathfrak{F}^{\prime}$ which is used in the proof. With this understanding the original proof becomes valid. I am grateful to $\mathrm{H}$. Tominaga for pointing this out (in a letter), and for a reference to his paper [2] in which the results of [1] are extended to primary rings. (Cf. another paper [3] by him in connection with [1] and [2].)

\section{REFERENCES}

1. Carl Faith, Galois extensions in which every element with regular trace is a normal basis element, Proc. Amer. Math. Soc. vol. 9 (1958) pp. 222-229.

2. H. Tominaga, A note on Galois theory of primary rings, Math. J. Okayama Univ. vol. 8 (1958) pp. 117-123.

3. - On normal basis theorem and strictly Galois extensions, Math. J. Okayama Univ. vol. 8 (1958) pp. 133-142.

Pennsylvania State University

Received by the editors September 24, 1959. 